\title{
FRANZ KRAPPEL
}

\section{Die Havanna Charta und die Entwicklung des Weltrohstoffhandels}

Berlin: Duncker \& Humblot 1975, 128 S., 29,60 DM

Bei dieser juristischen Dissertation handelt es sich um eine brauchbare Darstellung über die Entstehungsgeschichte, die Bestimmungen, schließlich das Scheitern und die Wiederbelebungsversuche der Havanna Charta und insbesondere des Kapitels VI über internationale Rohstoffabkommen sowie das GATT zu dieser Frage. Der dritte Teil ist der im ganzen ärgerliche Versuch, die Marktentwicklung und die intern. Warenabkommen für Zucker, Kaffee, Weizen, Olivenöl, Kakao und Zinn darzustellen und zu analysieren, die z. T. bis ins 19. Jahrhundert (mit ihren Vorläufern) zurückverfolgt wurden, aber alles in allem doch zu knapp ausfallen, um der realen und auch rechtlichen Entwicklung immer angemessen würdigen zu können. So feiert der Autor z. B. den Abschluß des Internationalen Kakaoabkommens 1972 als „wichtigen Fortschritt für die Entwicklung der Zusammenarbeit zwischen den Entwicklungsländern und den Industrieländern", wobei die Entwicklungsländer ihrem Ziel „Hilfe durch Handel zu ersetzen“ nähergekommen seien (S. 95). Bis heute ist nicht bekannt geworden, daß dieses (und auch das Nachfolgeabkommen) irgendeinen Einfluß auf die Preisentwicklung genommen hätte. Auch ist Krappel, der in den bestehenden Rohstoffabkommen eine „ungleiche Berücksichtigung von Erzeuger- und Verbraucherinteressen“ festzustellen meint, und damit den "ausgewogenen Rahmen der Havanna Charta" gesprengt sieht, offenbar entgangen, daß auch in den Rohstoffabkommen (gleichberechtigter) Bezug auf die Interessen von Produzenten und Konsumenten genommen wird. Dies entspricht auch weitgehend der Praxis, trotz aller diplomatischen Aktivitäten der Produzentenländer. Kartellbildungen (auf dem Rohstoffsektor), die gerade in diesem Zusammenhang in den Massenmedien immer wieder Schlagzeilen machen, werden nur am Rande behandelt.

Rolf Hanisch

\section{Diethard Mai:}

\section{Methoden sozialökonomischer Feldforschung - eine Einführung}

Materialien zur Reihe Sozialökonomische Schriften zur Agrarentwicklung, Hrsg. F. Kuhnen (Occasional Papers Bd. 6); Verlag der SSIP-Schriften, Saarbrücken 1976, 187 S., DM 7,一.

Die Arbeit unternimmt es, kurz und zusammenfassend in den Ablauf von Projekten empirischer Sozialforschung einzuführen. Nach einer Darstellung der hauptsächlichen erkenntnistheoretischen Positionen zur empirischen Sozialforschung wird der Entdeckungs-, Begründungs- und Verwertungszusammenhang von Forschungsprojekten erläutert und die Planung der Forschungskonzeption und der Feldarbeit dargelegt. Bei der Vorstellung der verschiedenen Methoden der Datenerhebung ist die durchgängige Verwendung eines neunstufigen Gliederungsschemas hervorzuheben, das die Übersicht erleichtert. Abschließend werden Fragen der Auswertung, Interpretation und Publikation von Forschungsergebnissen besprochen. Eine sehr ausführliche Zusammenstellung von übersetzten englischen Fachausdrücken der empirischen Sozialforschung dürfte insbesondere von Studienanfängern als nützlich empfunden werden. 